\title{
MULTISCALE SIMULATIONS OF POROUS MEDIA FLOWS IN FLOW-BASED COORDINATE SYSTEM
}

\author{
Y. EFENDIEV*, T. HOU ${ }^{\dagger}$, AND T. STRINOPOULOS ${ }^{\ddagger}$
}

\begin{abstract}
.
In this paper, we propose a multiscale technique for simulation of porous media flows in a flow-based coordinate system. Flow based coordinate system allows us to simplify the scale interaction and derive the upscaled equations for purely hyperbolic transport equations. We discuss the applications of the method to two-phase flows in heterogeneous porous media. For twophase flow simulations, the use of flow based coordinate system requires a limited global information, such as the solution of single-phase flow. Numerical results show that one can achieve accurate upscaling results using flow-based coordinate system.
\end{abstract}

1. Introduction. The modeling of two phase flow in porous formations is important for both environmental remediation and the management of petroleum reservoirs. Practical situations involving two phase flow include the dispersal of a non-aqueous phase liquid in an aquifer or the displacement of a non-aqueous phase liquid by water. In the subsurface, these processes are complicated by the effects of permeability heterogeneity on the flow and transport. Simulation models, if they are to provide realistic predictions, must accurately account for these effects. However, because permeability heterogeneity occurs at many different length scales, numerical flow models cannot in general resolve all of the scales of variation. Therefore, approaches are needed for representing the effects of subgrid scale variations on larger scale flow results. Typically, upscaled or multiscale models are employed for such systems. The main idea of upscaling techniques is to form coarse-scale equations with a prescribed analytical form that may differ from the underlying finescale equations. In multiscale methods, the fine-scale information is carried throughout the simulation and the coarse-scale equations are generally not expressed analytically, but rather formed and solved numerically.

On the fine (fully resolved) scale, the subsurface flow and transport of $N$ components can be described in terms of an elliptic (for incompressible systems) pressure equation coupled to a sequence of $N-1$ hyperbolic (in the absence of dispersive and capillary pressure effects) conservation laws. Our purpose in this paper is to perform upscaling of two-phase immiscible flow in a flow-based coordinate system. Flow-based coordinate system provides us with a solution that is better suited for upscaling because the solution is smoother and the scale interaction can be simpler. We would like to note that single-phase flow upscaling methods have been employed in the Cartesian framework using flow-based grids, [26]. In flow-based grid upscaling, the original equations are solved in a flow-based grid generated using the streamlines of the flow. Because of the similarities with the upscaling in flow-based grid, we use the terminology "flow-based coordinate system". The use of flow-based coordinate system allows us to perform accurate upscaling of transport equation which is purely hyperbolic. The upscaling of the transport equation along the streamlines in the current pressure-streamline coordinate system can be obtained since the equation is one dimensional. We discuss the upscaling of saturation equation across the streamlines. This type of upscaling introduces non-local macro-dispersion terms, where the macro-dispersion term involves two-point correlation of the velocity field along the streamlines. The advantage of using the flow-based coordinate system is that the computation of macrodispersion can be performed semi-analytically. This allows us to avoid typical difficulties ([14]) associated with the computation of the macrodiffusion term, including cross diffusion terms.

For upscaling of pressure equation, we employ multiscale finite element type methods. MsFEM is first introduced in [17]. Its main idea is to incorporate the small-scale information into finite element basis functions and capture their effect on the large scale via finite element computations. This approach shares common features with a number of other multiscale numerical methods, such as residual free bubbles [6,23], variational multiscale method [19], multiscale finite element method (MsFEM) [17], two-scale finite element methods [22], and two-scale conservative subgrid approaches [2]. We remark that special basis functions in finite element methods have been used earlier in [4] (cf. [3]). Multiscale finite element methodology has been modified and successfully applied to two-phase flow simulations in $[20,21,1]$ and later in [8].

\footnotetext{
*Department of Mathematics, Texas A\&M University, College Station, TX 77843-3368, email: efendiev@math.tamu.edu

${ }^{\dagger}$ Applied Mathematics, Caltech, Pasadena, CA 91125, email: hou@acm.caltech.edu

${ }^{\ddagger}$ Applied Mathematics, Caltech, Pasadena, CA 91125. email: theofilos@acm.caltech.edu
} 
Arbogast ([2]) used variational multiscale strategy and constructed a multiscale method for two-phase flow simulations. When considering two-phase flow upscaling, we use multiscale basis functions to compute the macrodispersion. Recently, a limited global information is used ([1], [11]) in constructing multiscale basis functions. It is interesting to note that in flow-based coordinate system these multiscale methods reduce to a standard multiscale finite element methods [17].

The paper is organized in the following way. In the next section we present the governing equations. In Section 3, we briefly present a motivation for our approach. Section 4 is devoted to the upscaling of transport equation (hyperbolic equation). In Section 5, we briefly mention multiscale finite element methods. The numerical results are presented in Section 6.

2. Fine-scale equations. We consider two-phase flow in a reservoir $\Omega$ under the assumption that the displacement is dominated by viscous effects; i.e., we neglect the effects of gravity, compressibility, and capillary pressure. Porosity will be considered to be constant. The two phases will be referred to as water and oil, designated by subscripts $w$ and $o$, respectively. We write Darcy's law, with all quantities dimensionless, for each phase as follows:

$$
\mathbf{v}_{j}=-\frac{k_{r j}(S)}{\mu_{j}} \mathbf{k} \cdot \nabla P
$$

where $\mathbf{v}_{j}$ is the phase velocity, $\mathbf{k}$ is the permeability tensor, $k_{r j}$ is the relative permeability to phase $j$ $(j=o, w), S$ is the water saturation (volume fraction), $P$ is pressure and $\mu_{j}$ is the viscosity of phase $j$ $(j=0, w)$. In this work, a single set of relative permeability curves is used and $\mathbf{k}$ is assumed to be a diagonal tensor. Combining Darcy's law with a statement of conservation of mass allows us to express the governing equations in terms of the so-called pressure and saturation equations:

$$
\begin{aligned}
& \nabla \cdot(\lambda(S) \mathbf{k} \cdot \nabla P)=q, \\
& \frac{\partial S}{\partial t}+\mathbf{v} \cdot \nabla f(S)=0
\end{aligned}
$$

where $\lambda$ is the total mobility, $f$ is the fractional flow of water, $q$ is a source term and $\mathbf{v}$ is the total velocity, which are respectively given by:

$$
\begin{gathered}
\lambda(S)=\frac{k_{r w}(S)}{\mu_{w}}+\frac{k_{r o}(S)}{\mu_{o}}, \quad f(S)=\frac{k_{r w}(S) / \mu_{w}}{k_{r w}(S) / \mu_{w}+k_{r o}(S) / \mu_{o}}, \\
\mathbf{v}=\mathbf{v}_{w}+\mathbf{v}_{o}=-\lambda(S) \mathbf{k} \cdot \nabla P .
\end{gathered}
$$

The above descriptions are referred to as the fine model of the two-phase flow problem. For simplicity, in further analysis we will assume $q=0$ and impose non-homogeneous boundary conditions.

For simplicity, in further analysis we will assume $q=0$.

3. Motivation. The upscaling and multiscale methods for two-phase flow systems are discussed by many authors. In most upscaling procedures, the upscaled quantities are computed on a coarse grid obtained from the underlying fine-grid. Because of strong scale interactions associated with complex spatial correlations, most upscaling and multiscale methods may require some type of global information or border regions to take into account non-local neighboring information. In the proposed work, multiscale and upscaling techniques are used in a flow-based coordinate system. This coordinate system, which is based on a limited global information, can provide additional smoothness for the solution and simplifies the scale interaction.

Next, we present the flow-based coordinate system and the modified equation and discuss some asymptotic properties of the modified equations in the flow-based coordinate system. We will restrict our analysis to the two-dimensional case and assume that the heterogeneous porous medium is isotropic, $\mathbf{k}(\mathbf{x})=k(\mathbf{x}) \mathbf{I}$. 
In general the stream function is defined as $\nabla \times \psi=\mathbf{v}=\left(v_{1}, v_{2}\right)$. In two dimensions the stream function $\psi$ reduces to a scalar field defined by

$$
\partial \psi / \partial x_{1}=-v_{2}, \quad \partial \psi / \partial x_{2}=v_{1} .
$$

It can be easily shown that $\nabla \psi \cdot \nabla p=0$, where $p$ is initial pressure. Denote the initial stream function and pressure by $\psi=\psi(x, t=0)$ and $p=P(x, t=0)$. If we assume $S=0$ at time zero, then $(\psi, p)$ can be obtained from the pressure equation with $\lambda(S)=1$. Then the equation for pressure and stream function can be written down in this curvilinear orthogonal coordinate system (see [11], [24])

$$
\frac{\partial}{\partial \psi}\left(k^{2} \lambda(S) \frac{\partial P}{\partial \psi}\right)+\frac{\partial}{\partial p}\left(\lambda(S) \frac{\partial P}{\partial p}\right)=0 .
$$

Applying the same change of variables to saturation equation, we get

$$
\frac{\partial S}{\partial t}+(\mathbf{v} \cdot \nabla \psi) \frac{\partial f(S)}{\partial \psi}+(\mathbf{v} \cdot \nabla p) \frac{\partial f(S)}{\partial p}=0 .
$$

Our objective is to present an upscaling method in pressure-streamline framework for two-phase flow equations. The cornerstone of our upscaling method is the fact that the pressure at later times is a smooth function of initial pressure and thus the upscaling of initial pressure-streamline framework is more robust and accurate. In [11], the authors consider a special case when the permeability has strong non-local effects. The authors show that in this case the pressure evolution can be written as

$$
P(\psi, p, t)=P_{0}(p, t)+\text { h.o.t }
$$

where $P_{0}$ is the solution of

$$
\frac{\partial}{\partial p}\left(\lambda_{0}(p, t) \frac{\partial P_{0}}{\partial p}\right)=0,
$$

where $\lambda_{0}$ depends $S_{0}$. Using (3.4) one can show

$$
S(\psi, p, t)=S_{0}(p, t)+\text { h.o.t. }
$$

This expansion shows that in porous media with strong channelized non-local effects, the initial pressurestreamline coordinate system can provide a better coordinate system for performing upscaling. First, this coordinate system can simplify the scale interaction and non-local effects can be modeled more accurately.

\section{Upscaling of saturation equation in flow-based coordinate system.}

4.1. Homogenization of saturation equation. In this section, we would like to derive an upscaled model for the transport equation. We will assume the velocity is independent of time, $\lambda(S)=1$, and restrict ourselves to the two-dimensional case. Then using the pressure-streamline framework, one obtains

$$
\begin{aligned}
S_{t}^{\epsilon}+v_{0}^{\epsilon} f\left(S^{\epsilon}\right)_{p} & =0 \\
S(p, \psi, t=0) & =S_{0},
\end{aligned}
$$

where $\epsilon$ denotes the small scale and $v_{0}^{\epsilon}$ denotes the Jacobian of the transformation and is positive. For simplicity, we assume $\mathbf{k}(\mathbf{x})=k(\mathbf{x}) \mathbf{I}$ and we have $\nabla \psi \cdot \nabla p=0$. For deriving upscaled equations, we will first homogenize (4.1) along the streamlines, and then to homogenize across the streamlines. The homogenization along the streamlines can be done following Bourgeat and Mikelic [5] or following Hou and Xin [18] and E [10]. The latter uses two-scale convergence theory and we refer to [24] for the results on homogenization of (4.1) using two-scale convergence theory. We note that the homogenization results of Bourgeat and Mikelic is for general heterogeneities without an assumption on periodicity, and thus, is more appropriate for problems considered in the paper. Following [5], the homogenization of (4.1) can be easily derived (see Proposition 3.4 in $[5])$. 
For ease of notations, we ignore the $\psi$ dependence of $v_{0}^{\epsilon}$ and $S^{\epsilon}$, and treat $\psi$ as a parameter. We consider

$$
v_{0}^{\epsilon}(p)=v_{0}\left(p, \frac{p}{\epsilon}\right) .
$$

Moreover, we assume that the domain is a unit interval. Then, for each $\psi$, it can be shown that $S^{\epsilon}(p, \psi, t) \rightarrow$ $\tilde{S}(p, \psi, t)$ in $L^{1}((0,1) \times(0, T))$, where $\tilde{S}$ satisfies

$$
\tilde{S}_{t}+\tilde{v}_{0} f(\tilde{S})_{p}=0
$$

and where $\tilde{v}_{0}$ is harmonic average of $v_{0}^{\epsilon}$, i.e.,

$$
\frac{1}{v_{0}^{\epsilon}} \rightarrow \frac{1}{\tilde{v}_{0}} \quad \text { weak } * \text { in } L^{\infty}(0,1) .
$$

The proof of this fact follows from Proposition 3.4. of [5]. Here, we briefly sketch the proof.

Following [5] and assuming for simplicity $\int_{0}^{1} \frac{d \eta}{v_{0}^{\epsilon}(\eta)}=\int_{0}^{1} \frac{d \eta}{\tilde{v}_{0}(\eta)}=1$, we introduce

$$
\frac{d X^{\epsilon}(p)}{d p}=v_{0}^{\epsilon}\left(X^{\epsilon}(p)\right), \quad \frac{d X^{0}(p)}{d p}=\tilde{v}_{0}\left(X^{0}(p)\right) .
$$

Then (Lemma 3.1 of [5]):

$$
X^{\epsilon} \rightarrow X^{0} \quad \text { in } C[0,1] \text { as } \epsilon \rightarrow 0 .
$$

Consequently,

$$
\begin{array}{r}
\int_{0}^{T} \int_{0}^{1}\left|S^{\epsilon}(p, \tau)-\tilde{S}(p, \tau)\right| d p d \tau=\int_{0}^{T} \int_{0}^{1}\left|S^{\epsilon}\left(X^{\epsilon}(p), \tau\right)-\tilde{S}\left(X^{\epsilon}(p), \tau\right)\right| v_{0}^{\epsilon}\left(X^{\epsilon}(p)\right) d p d \tau \leq \\
\int_{0}^{T} \int_{0}^{1}\left|S^{\epsilon}\left(X^{\epsilon}(p), \tau\right)-\tilde{S}\left(X^{0}(p), \tau\right)\right| v_{0}^{\epsilon}\left(X^{\epsilon}(p)\right) d p d \tau+ \\
\int_{0}^{T} \int_{0}^{1}\left|\tilde{S}\left(X^{\epsilon}(p), \tau\right)-\tilde{S}\left(X^{0}(p), \tau\right)\right| v_{0}^{\epsilon}\left(X^{\epsilon}(p)\right) d p d \tau \leq \\
\int_{0}^{T} \int_{0}^{1}\left|S^{\epsilon}\left(X^{\epsilon}(p), \tau\right)-\tilde{S}\left(X^{0}(p), \tau\right)\right| d p d \tau+\int_{0}^{T} \int_{0}^{1}\left|\tilde{S}\left(X^{\epsilon}(p), \tau\right)-\tilde{S}\left(X^{0}(p), \tau\right)\right| d p d \tau
\end{array}
$$

The first term on the right hand side of (4.4) converges to zero because $S^{\epsilon}\left(X^{\epsilon}(p), \tau\right)$ and $\tilde{S}\left(X^{0}(p), \tau\right)$ satisfy the same equation $u_{t}+f(u)_{p}=0$, however, with the following initial conditions $S^{\epsilon}\left(X^{\epsilon}(p), t=0\right)=S_{0} \circ X^{\epsilon}$ $\tilde{S}\left(X^{0}(p), \tau\right)=S_{0} \circ X^{0}$. Because of (4.3) and comparison principle

$$
\int_{0}^{T} \int_{0}^{1}\left|S^{\epsilon}\left(X^{\epsilon}(p), \tau\right)-\tilde{S}\left(X^{0}(p), \tau\right)\right| d p d \tau \leq C \int_{0}^{1}\left|S_{0} \circ X^{\epsilon}-S_{0} \circ X^{0}\right| d p,
$$

the first term converges to zero. The convergence of the second term for each $\psi$ follows from the argument in [5] (page 368) using Lebesgue's dominated convergence theorem.

Next, we provide a convergence rate (see also [24]) of the fine saturation $S^{\epsilon}$ to the homogenized limit $\tilde{S}$ as $\epsilon \rightarrow 0$.

TheOrem 4.1. Assume that $v_{0}^{\epsilon}(p)$ is bounded uniformly

$$
C^{-1} \leq v_{0}^{\epsilon}\left(p, \frac{p}{\epsilon}\right) \leq D
$$

Denote by $F(t, T)$ the solution to $S_{t}+f(S)_{T}=0$. The solution $\tilde{S}$ of (4.2) converges to $S^{\epsilon}$ (assuming initial conditions that don't depend on the fast scale) at a rate given by 
TABLE 4.1

Numerical demonstration of Theorem 4.1

\begin{tabular}{|c||c|c|c|c|c|c|c|}
\hline & 2 & 4 & 8 & 16 & 32 & 64 & 128 \\
\hline \hline$L_{1}$ & 0.0432 & 0.0124 & 0.0063 & 0.0049 & 0.0022 & $6.62 \times 10^{-4}$ & $1.41 \times 10^{-4}$ \\
\hline$L_{\infty}$ & 0.670 & 0.664 & 0.663 & 0.665 & 0.653 & 0.61 & 0.096 \\
\hline
\end{tabular}

$\left\|S^{\epsilon}-\tilde{S}\right\|_{\infty} \leq G \epsilon$

when $F$ remains Lipschitz for all time, and

$$
\left\|S^{\epsilon}-\tilde{S}\right\|_{n} \leq G \epsilon^{1 / n}
$$

when $F$ develops at most a finite number of discontinuities.

The proof of this theorem is provided in Appendix (see also [24]). We briefly show the results of a numerical experiment to demonstrate the estimate of Theorem 4.1 for a discontinuous solution. To find the rate of convergence of $\tilde{S}$ to $S^{\epsilon}$ we have to use a grid that resolves the velocity and the shock so that numerical error, especially the numerical diffusion near the shock, does not mask the upscaling error. At the same time the velocity must vary enough in the cells so that the upscaling error is large. To avoid numerical diffusion we use a small final time. We consider a nonlinear flux with $\mu_{o}=\mu_{w}=1, T_{\text {final }}=0.1$, $v=1-20 p \sin \left(10 \pi \frac{1}{p+0.1}\right)+20 \sin \left(5 \frac{\pi}{2}\right)$. We used a grid of 4096 cells for both upscaled and fine computation. The upscaling error as a function of the number of averaging cells is shown in Table 4.1. The $L_{\infty}$ norm shows that in all experiments with less than 64 averaging cells the numerical diffusion was not significant. The convergence rate seems to be slightly larger than 1, which is consistent with theorem 4.1.

The homogenized operator given by (4.2) still contains variation of order $\epsilon$ through the fast variable $\frac{\psi}{\epsilon}$, however there it does not contain any derivatives in that variable. Its dependence on $\frac{\psi}{\epsilon}$ is only parametric. We can homogenize the dependence of the partially homogenized operator on $\frac{\psi}{\epsilon}$ and arrive at a homogenized operator that is independent of the small scale. In the latter case, we will only obtain weak convergence of the partially homogenized solution. When we homogenized along the streamlines, the resulting equation was of hyperbolic type like the original equation. In a seminal and celebrated paper, Tartar [25] showed that homogenization across streamlines leads to transport with the average velocity plus a time-dependent diffusion term, referred to as macrodispersion, a physical phenomenon that was not present in the original fine equation. In particular, if the velocity field does not depend on $p$ inside the cells, that is, $\tilde{v}\left(\psi, \frac{\psi}{\epsilon}\right)$, then the homogenized solution, $\bar{S}$, (weak* limit of $\tilde{S}$, which will be denoted by $\bar{S}$ ), satisfies

$$
\bar{S}_{t}+\overline{\tilde{v}}_{0} \bar{S}_{p}=\int_{0}^{t} \int \bar{S}_{p p}(p-\lambda(t-\tau), \psi, \tau) d \mu_{\frac{\psi}{\epsilon}}(\lambda) d \tau .
$$

Here, $d \nu_{\frac{\psi}{\epsilon}}$ the Young measure associated with the sequence $\tilde{v}_{0}(\psi, \cdot)$ and $d \mu_{\frac{\psi}{\epsilon}}$ is a Young measure that satisfies

$$
\left(\int \frac{d \nu_{\frac{\psi}{\epsilon}}(\lambda)}{\frac{s}{2 \pi i q}+\lambda}\right)^{-1}=\frac{s}{2 \pi i q}+\overline{\tilde{v}}_{0}-\int \frac{d \mu_{\frac{\psi}{\epsilon}}(\lambda)}{\frac{s}{2 \pi i q}+\lambda} .
$$

We have denoted by $\bar{v}_{0}$ the weak limit of the velocity. This equation has no dependence on the small scale and we consider it to be the full homogenization of the fine saturation equation. Efendiev and Popov [15] have extended this method for the Riemann problem in the case of nonlinear flux. Note that the homogenization across streamlines provide a weak limit of partially homogenized solution. Because the original solution $S^{\epsilon}$ strongly converges to partially homogenized solution for each $\psi$, it can be easily shown that $S^{\epsilon} \rightarrow \bar{S}$ weakly. We omit this proof here. 
In numerical simulations, it is difficult to use (4.5) as a homogenized operator, and often a second order approximation of this equation is used. These approximate equations can be also derived using perturbation analysis. In particular, using the higher moments of the saturation and the velocity, one can model the macrodispersion. In the context of two-phase flow this idea was introduced by Efendiev, Durlofsky, and Lee $[14,13]$. In our case, the computation of the macrodispersion is much simpler because the transport equations have been already averaged along the streamlines, and thus we will be applying perturbation technique to one dimensional problem.

We expand $\tilde{S}, \tilde{v_{0}}$ (following [14]) as an average over the cells in the pressure-streamline frame and the corresponding fluctuations

$$
\begin{aligned}
\tilde{S} & =\bar{S}(p, \psi, t)+S^{\prime}(p, \psi, \zeta, t) \\
\tilde{v}_{0} & =\tilde{v}_{0}(p, \psi, t)+\tilde{v}_{0}^{\prime}(p, \psi, \zeta, t) .
\end{aligned}
$$

We will derive the homogenized equation for $f(S)=S$. Averaging equations (4.2) with respect to $\psi$ we find an equation for the mean of the saturation

$$
\bar{S}_{t}+{\overline{\tilde{v}_{0}}}_{p}+\overline{\tilde{v}_{0}^{\prime} S_{p}^{\prime}}=0 .
$$

An equation for the fluctuations is obtained by subtracting the above equation from (4.2)

$$
S_{t}^{\prime}+\left(\tilde{v}_{0}-\overline{\tilde{v}_{0}}\right) \bar{S}_{p}+\tilde{v}_{0} S_{p}^{\prime}-\overline{\tilde{v}_{0}^{\prime} S_{p}^{\prime}}=0 .
$$

Together, the equations for the saturation are

$$
\begin{array}{r}
\bar{S}_{t}+{\overline{\tilde{v}_{0}}}_{p}+\overline{\tilde{v}_{0}^{\prime} S_{p}^{\prime}}=0 \\
S_{t}^{\prime}+\tilde{v}_{0}^{\prime} \bar{S}_{p}+\tilde{v}_{0} S_{p}^{\prime}-\overline{\tilde{v}_{0}^{\prime} S_{p}^{\prime}}=0 .
\end{array}
$$

We can consider the second equation to be the auxiliary (cell) problem and the first equation to be the upscaled equation. We remind that the cell problem for a hyperbolic equation is $O(1)$ whereas for an elliptic it is $O(\epsilon)$. We can obtain an approximate numerical method by solving the cell problem only near the shock region in space time, where the macrodispersion term is largest. In that case it is best to diagonalize these equations by adding the first to the second one

$$
\begin{array}{ccc}
\bar{S}_{t}+\overline{\tilde{v}}_{0} \bar{S}_{p} & = & -\overline{\tilde{v}_{0}^{\prime}\left(\tilde{S}_{p}-\bar{S}_{p}\right)} \\
\tilde{S}_{t}+\tilde{v}_{0} \tilde{S}_{p} & = & 0 .
\end{array}
$$

Compared to (4.7), it has fewer forcing terms and no cross fluxes, which leads to a numerical method with less numerical diffusion that is easier to implement. In most cases we can make a better approximation that is described in the next sections.

4.2. Comparison with the Cell Problem in the Cartesian frame. Before we derive the numerical approximation we will briefly compare the homogenized equations in flow-based frame with the homogenized equations in a Cartesian frame obtained recently in [16], [27]. The homogenized equations in the Cartesian variables as derived in [16], [27] are defined in terms of the average saturation over the coarse blocks $\bar{S}$ and the fluctuations $S^{\prime \prime}$. Note that whereas the fluctuations $S^{\prime}$ in the pressure-streamline frame depend only on one fast variable, the fluctuations $S^{\prime \prime}$ in the Cartesian frame depend on two fast variables. $\mathcal{P}$ is a projection operator onto the average along the streamlines within the cell, which corresponds to the fast variable along the streamlines and $\mathcal{Q}$ is a projection onto the orthogonal complement so that any function $u$ can be written as $u=\mathcal{P}(u)+\mathcal{Q}(u)$. With this notation the homogenized equations are

$$
\begin{array}{ccc}
\bar{S}_{t}+\overline{\mathbf{v}} \cdot \nabla \bar{S}+\nabla \cdot \overline{\mathbf{v}^{\prime \prime} S^{\prime \prime}} & = & 0 \\
S_{t}^{\prime \prime}+\left(\overline{\mathbf{v}}+\mathcal{P}\left(\mathbf{v}^{\prime \prime}\right)\right) \cdot \nabla S^{\prime \prime}+\mathcal{P}\left(\mathbf{v}^{\prime \prime}\right) \cdot \nabla \bar{S}-\nabla \cdot \overline{\mathbf{v}^{\prime \prime} S^{\prime \prime}} & = & G\left(x, \frac{x}{\epsilon}, t\right), \\
6 &
\end{array}
$$


where

$$
G\left(x, \frac{x}{\epsilon}, t\right)=\left(\overline{\mathbf{v}}+\mathcal{P}\left(\mathbf{v}^{\prime \prime}\right)\right) \cdot \mathcal{Q}\left(\nabla S^{\prime \prime}\right)-\mathcal{P}\left(\mathcal{Q}\left(\mathbf{v}^{\prime \prime}\right) \cdot \mathcal{Q}\left(\nabla S^{\prime \prime}\right)\right)+\mathcal{Q}\left(S^{\prime \prime}\right) .
$$

The Cartesian cell problem, which is the equation for the fluctuations in (4.8), is a two dimensional equation along two fast variables. Prior to solving it, one must compute the projections $\mathcal{P}$ and $\mathcal{Q}$ which adds to the complexity of the method and its computational cost. In contrast the pressure-streamline cell problem in equations (4.7) contains only one fast variable and no projection operator. In some sense, in the pressure-streamline frame the projection operation, which was carried out by restricting the oscillatory test functions, removes a fast variable and reduces one fast dimension to arrive at the cell problem of (4.7). In the Cartesian frame the projection operation remains in the equations in the form of $\mathcal{P}$ and $\mathcal{Q}$ and the fast variation along the flow is not cleanly removed. This is a strong indication that the pressure-streamline frame reveals the structure of the flow correctly.

4.3. Numerical Averaging across Streamlines. The derivation in the previous sections contained no approximation. In this section, we follow the same idea as in the derivation to solve the equation for the fluctuations along the characteristics, but with the purpose of deriving an equation on the coarse grid. To achieve this, we will not perform analytical upscaling in the sense of deriving a continuous upscaled equation as in the previous section. We will first discretize the equation with a finite volume method in space and then upscaled the resulting equation. Our upscaled equation will therefore be dependent on the numerical scheme.

We use the same definition for the average saturation and the fluctuations as in (4.6) and follow the same steps until equation (4.7). We discretize the macrodispersion term in the equation for the average saturation

$$
\overline{\tilde{v}_{0}^{\prime} S_{p}^{\prime}}=\frac{{\overline{\tilde{v}_{0}^{\prime} S^{\prime}}}^{i+1}-{\overline{\tilde{v}_{0}^{\prime} S^{\prime}}}^{i}}{\Delta p}+O(\Delta p)
$$

A superscript ${ }^{i}$ refers to a discrete quantity defined at the center of the conservation cell. Instead of solving the equation for the fluctuations on the fine characteristics as before, which would lead to a fine grid algorithm, we solve it on the coarse characteristics defined by

$$
\frac{d P}{d t}=\overline{\tilde{v}_{0}}, \text { with } P(p, 0)=p
$$

Compared to the equation that we obtained in the previous section for $S^{\prime}$, this equation for $S^{\prime}$ has an extra term, which appears second

$$
\left.S^{\prime}=-\int_{0}^{t}\left(\tilde{v}_{0}^{\prime}(P(p, \tau), \psi) \bar{S}_{p}(P(p, \tau), \psi, \tau)+\tilde{v}_{0}^{\prime}(P(p, \tau), \psi) S_{p}^{\prime}(P(p, \tau), \psi, \tau)+\overline{\tilde{v}_{0}^{\prime} S_{p}^{\prime}}\right)\right) d \tau
$$

The second term is second-order in fluctuating quantities, and we expect it to be smaller than the first term so we neglect it. As before, we multiply by $\tilde{v}_{0}^{\prime}$ and average over $\psi$ to find

$$
\overline{\tilde{v}_{0}^{\prime} S^{\prime}}=-\int_{0}^{t} \overline{\tilde{v}_{0}^{\prime} \tilde{v}_{0}(P(p, \tau), \psi) \bar{S}_{p}(P(p, \tau), \psi, \tau)} d \tau .
$$

In this form at time $t$ it is necessary to know information about the past saturation in $(0, t)$ to compute the future saturation. Following [14], it can be easily shown that $\bar{S}_{p}(P(p, \tau)$ depends weakly on time, in the sense that the difference between $\bar{S}_{p}\left(P(p, \tau)\right.$ and $\bar{S}_{p}(P(p, t)$ is of third-order in fluctuating quantities. Therefore we can take $\bar{S}_{p}(P(p, \tau)$ out of the time integral to find

$$
\overline{\tilde{v}_{0}^{\prime} S^{\prime}}=-\int_{0}^{t} \overline{\tilde{v}_{0}^{\prime} \tilde{v}_{0}^{\prime}(P(p, \tau), \psi)} d \tau \bar{S}_{p} .
$$


The term inside the time integral is the covariance of the velocity field along each streamline. The macrodispersion in this form can be computed independent of the past saturation.

The nonlinearity of the flux function introduces an extra source of error in the approximation. We expand $f(\tilde{S})$ near $\bar{S}$ (cf. [13]) and keep only the first term

$$
\begin{gathered}
\tilde{S}=\bar{S}(p, \psi, t)+S^{\prime}(p, \psi, \zeta, t) \\
\tilde{v}_{0}=\tilde{v}_{0}(p, \psi, t)+\tilde{v}_{0}^{\prime}(p, \psi, \eta, t) \\
\bar{f}(\tilde{S})=f(\bar{S})+f_{S}(\bar{S}) S^{\prime}+O\left(S^{\prime 2}\right) \\
f(S)_{p}=f_{S}(\bar{S}) \bar{S}_{p}+f(\bar{S}) S^{\prime}+\ldots
\end{gathered}
$$

This approximation is not accurate near the shock because $S^{\prime}$ is not small near sharp fronts. The region near the shock is important because the macrodispersion is large. Due to the dependence of the jump in the saturation on the mobility we expect this approximation to be better for lower mobilities. Nevertheless this approximation works well in practice. For more accuracy, it is also possible to retain more terms in the Taylor expansion. We will show that in realistic examples these higher-order terms are not important in our setting.

Using these definitions we derive the following equations for the average saturation and the fluctuations (see [24] for more details)

$$
\begin{array}{r}
\bar{S}_{t}+\overline{\tilde{v}_{0}} f(\bar{S})_{p}+\overline{\tilde{v}_{0}^{\prime}\left(f_{S}(\bar{S}) S^{\prime}\right)_{p}}=0 \\
S_{t}^{\prime}+\tilde{v}_{0}^{\prime} f_{S}(\bar{S}) \bar{S}_{p}+\tilde{v}_{0} f_{S}(\bar{S}) S_{p}^{\prime}-\overline{\tilde{v}_{0}^{\prime} S_{p}^{\prime}}=0 .
\end{array}
$$

The macrodispersion is discretized as

$$
\overline{\tilde{v}_{0}^{\prime}\left(f_{S}(\bar{S}) S^{\prime}\right)_{p}}=\frac{{\overline{\tilde{v}_{0}^{\prime} f_{S}(\bar{S}) S^{\prime}}}^{i+1}-{\overline{\tilde{v}_{0}^{\prime} f_{S}(\bar{S}) S^{\prime}}}^{i}}{\Delta p}+O(\Delta p) .
$$

We solve the second equation on the coarse characteristics defined by

$$
\frac{d P}{d t}=\overline{\tilde{v}_{0}} f_{S}(\bar{S}) \text {, with } P(p, 0)=p
$$

and form the terms that appear in the macrodispersion

$$
\overline{\tilde{v}_{0}^{\prime} f_{S}(\bar{S}) S^{\prime}}=-\int_{0}^{t} \overline{\tilde{v}_{0}^{\prime} f_{S}(\bar{S}) \tilde{v}_{0}^{\prime}(P(p, \tau), \psi) f_{S}(\bar{S}(P(p, \tau), \psi, \tau)) \bar{S}_{p}(P(p, \tau), \psi, \tau)} d \tau .
$$

As before we have dropped terms that are second-order in fluctuating quantities. It can be shown (see [24]) that $f_{S}(\bar{S}(P(p, \tau), \psi, \tau)) \bar{S}_{p}(P(p, \tau), \psi, \tau)$ does not vary significantly along the streamlines and it can be taken out of the integration in time:

$$
\overline{\tilde{v}_{0}^{\prime} f_{S}(\bar{S}) S^{\prime}}=-\int_{0}^{t} \overline{\tilde{v}_{0}^{\prime} \tilde{v}_{0}^{\prime}(P(p, \tau), \psi)} d \tau f_{S}(\bar{S})^{2} \bar{S}_{p} .
$$

This expression is similar to the one obtained in the linear case, however the macrodispersion depends on the past saturation through the equation for the coarse characteristics.

Even though the macrodispersion depends on the past saturation it is possible to compute it incrementally as it is done in [13]. Given its value $D(t)$ at time $t$ we compute the values at $t+\Delta t$ using the macrodispersion at the previous time

$$
D(t+\Delta t)=\int_{0}^{t+\Delta t} \ldots d \tau=\int_{0}^{t} \ldots d \tau+\int_{t}^{t+\Delta t} \ldots d \tau .
$$

This is possible because in the derivation for the approximate expression for the macrodispersion we took the terms that depend on $S(\tau)$ outside the time integration. The integrand, the average covariance of the velocity field along the streamlines, needs to be computed only once at the beginning. Then updating the macrodispersion takes $O\left(n^{2}\right)$ computations, as many as it takes to update $\bar{S}$. 
5. Multiscale finite element methods for upscaling of pressure equation. The multiscale finite element framework will be used for upscaling of pressure equation. We will choose a finite volume element method as a global solver. We choose a finite volume method because, by its construction, it satisfies local conservation on the discrete level, which is important in groundwater and reservoir simulations. The proposed method is similar to earlier introduced multiscale finite volume method in $[20,21]$. Let $\mathcal{K}^{h}$ denote the collection of coarse elements/rectangles $K$. Consider a coarse element $K$, and let $\boldsymbol{\xi}_{K}$ be its center. The element $K$ is divided into four rectangles of equal area by connecting $\boldsymbol{\xi}_{K}$ to the midpoints of the element's edges. We denote these quadrilaterals by $K_{\boldsymbol{\xi}}$, where $\boldsymbol{\xi} \in Z_{h}(K)$, are the vertices of $K$. Also, we denote $Z_{h}=\bigcup_{K} Z_{h}(K)$ and $Z_{h}^{0} \subset Z_{h}$ the vertices which do not lie on the Dirichlet boundary of $\Omega$. The control volume $V_{\boldsymbol{\xi}}$ is defined as the union of the quadrilaterals $K_{\boldsymbol{\xi}}$ sharing the vertex $\boldsymbol{\xi}$.

The key idea of the method is the construction of basis functions on the coarse grids, such that these basis functions capture the small-scale information on each of these coarse grids. The method that we use follows its finite element counterpart presented in [17]. The basis functions are constructed from the solution of the leading order homogeneous elliptic equation on each coarse element with some specified boundary conditions. Thus, if we consider a coarse element $K$ that has $d$ vertices, the local basis functions $\phi_{i}, i=1, \cdots, d$ are set to satisfy the following elliptic problem:

$$
\begin{aligned}
-\nabla \cdot\left(\mathbf{k} \cdot \nabla \phi_{i}\right) & =0 \quad \text { in } K \\
\phi_{i} & =g_{i} \quad \text { on } \partial K,
\end{aligned}
$$

for some function $g_{i}$ defined on the boundary of the coarse element $K$. Hou et al. [17] have demonstrated that a careful choice of boundary conditions would improve the accuracy of the method. In previous findings, the function $g_{i}$ for each $i$ is chosen to vary linearly along $\partial K$ or to be the solution of the local one-dimensional problems [20] or the solution of the problem in a slightly larger domain is chosen to define the boundary conditions. The boundary conditions for the basis functions that are used in this paper will be discussed later. We will require $\phi_{i}\left(\mathbf{x}_{j}\right)=\delta_{i j}$. Finally, a nodal basis function associated with the vertex $\mathbf{x}_{i}$ in the domain $\Omega$ are constructed from the combination of the local basis functions that share this $\mathbf{x}_{i}$ and zero elsewhere. We would like to note that one can use an approximate solution of (5.1) when it is possible. For example, in the case of periodic or scale separation cases, the basis functions can be approximated using homogenization expansion (see [12]). This type of simplification is not applicable for problems considered in this paper.

Next, we denote by $V^{h}$ the space of our approximate pressure solution, which is spanned by the basis functions $\left\{\phi_{j}\right\}_{\mathbf{x}_{j} \in Z_{h}^{0}}$. Then we formulate the finite dimensional problem corresponding to finite volume element formulation of (2.2). A statement of mass conservation on a coarse-control volume $V_{\mathbf{x}}$ is formed from (2.2), where now the approximate solution is written as a linear combination of the basis functions. Assembly of this conservation statement for all control volumes would give the corresponding linear system of equations that can be solved accordingly. The resulting linear system has incorporated the fine-scale information through the involvement of the nodal basis functions on the approximate solution. To be specific, the problem now is to seek $P^{h} \in V^{h}$ with $P^{h}=\sum_{\mathbf{x}_{j} \in Z_{h}^{0}} P_{j} \phi_{j}$ such that

$$
\int_{\partial V_{\xi}} \lambda(S) \mathbf{k} \cdot \nabla P^{h} \cdot \mathbf{n} d l=0
$$

for every control volume $V_{\boldsymbol{\xi}} \subset \Omega$. Here $\vec{n}$ defines the normal vector on the boundary of the control volume, $\partial V_{\boldsymbol{\xi}}$ and $S$ is the fine-scale saturation field at this point. We note that concerning the basis functions, a vertex-centered finite volume difference is used to solve (5.1), and using the harmonic average to approximate the permeability $\mathbf{k}$ at the edges of fine control volumes.

In the previous studies $[1,11,7]$, it was found that the use of global information can improve the multiscale finite element method. In particular, the solution of the pressure equation at initial time is used to construct the boundary conditions for the basis functions. It is interesting to note that the multiscale finite element methods that employ a limited global information reduces to standard multiscale finite element method in flow-based coordinate system. This can be verified directly and the reason behind it is that we have already employed a limited global information in flow-based coordinate system. 

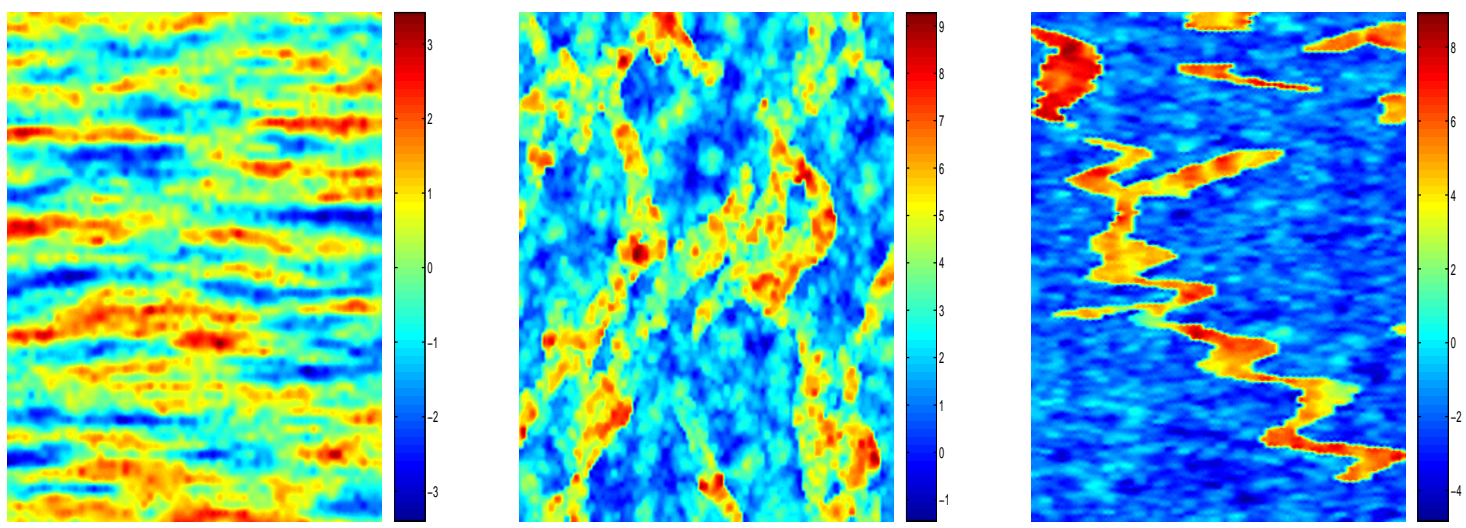

FIG. 6.1. Permeability fields used in the simulations. Left - permeability field with exponential variogram, middle synthetic channelized permeability field, right - layer 36 of SPE comparative project [9]

6. Numerical Results. In this section, we first show representative simulation results for $\lambda(S)=1$ for flux functions $f(S)=S$ and nonlinear $f(S)$ with viscosity ratio $\mu_{o} / \mu_{w}=5$. For such setting, the pressure and saturation equations are decoupled and we can investigate the accuracy of saturation upscaling independently from the pressure upscaling. At the end of the section we will present numerical results for two-phase flow. We consider two type of permeability fields. The first type includes a permeability field generated using two-point geostatistics with correlation lengths $l_{x}=0.3, l_{z}=0.03$ and $\sigma^{2}=1.5$ (see Figure 6.1 , left). The second type of permeability fields correspond to a channelized system, and we consider two examples. The first example (middle figure of Figure 6.1) is a synthetic channelized reservoir generated using both multi-point geostatistics (for the channels) and two-point geostatistics (for permeability distribution within each facies). The second channelized system is one of the layers of the benchmark test (representing the North Sea reservoir), the SPE comparative project [9] (upper Ness layers). These permeability fields are highly heterogeneous, channelized, and difficult to upscale. Because the permeability fields are highly heterogeneous, they are refined to $400 \times 400$ in order to obtain accurate comparisons.

Simulation results will be presented for saturation snapshots as well as the oil cut as a function of pore volume injected (PVI). Note that the oil cut is also referred to as the fractional flow of oil. The oil cut (or fractional flow) is defined as the fraction of oil in the produced fluid and is given by $q_{o} / q_{t}$, where $q_{t}=q_{o}+q_{w}$, with $q_{o}$ and $q_{w}$ being the flow rates of oil and water at the production edge of the model. In particular, $q_{w}=\int_{\partial \Omega^{\text {out }}} f(S) \mathbf{v} \cdot \mathbf{n} d l, q_{t}=\int_{\partial \Omega^{\text {out }}} \mathbf{v} \cdot \mathbf{n} d l$, and $q_{o}=q_{t}-q_{w}$, where $\partial \Omega^{\text {out }}$ is the outer flow boundary. We will use the notation $Q$ for total flow $q_{t}$ and $F$ for fractional flow $q_{o} / q_{t}$ in numerical results. Pore volume injected, defined as $P V I=\frac{1}{V_{p}} \int_{0}^{t} q_{t}(\tau) d \tau$, with $V_{p}$ being the total pore volume of the system, provide a dimensionless time for the displacement.

When using multiscale finite element methods for two-phase flow, one can update the basis functions near the sharp fronts. Indeed, sharp fronts modify the local heterogeneities and this can be taken into account by re-solving the local equations, (5.1), for basis functions. If the saturation is smooth in the coarse block, it can be approximated by its average in (5.1), and consequently, the basis functions do not needed to be updated. It can be shown that this approximation yields first-order errors (in terms of coarse mesh size). In our simulations, we have found only a slight improvement when the basis functions are updated, thus the numerical results for the MsFVEM presented in this paper do not include the basis function update near the sharp fronts. Since a pressure-streamline coordinate system is used the boundary conditions are given by $P=1, S=1$ along the $p=1$ edge and $P=0$ along the $p=0$ edge, and no flow boundary condition on the rest of the boundaries.

For the upscaled saturation equation, which is a convection-diffusion equation, we need to observe an 
TABLE 6.1

Upscaling error for permeability generated using two-point geostatistics

\begin{tabular}{|c||c|c|c|c|}
\hline LINEAR FLUX & $25 \times 25$ & $50 \times 50$ & $100 \times 100$ & $200 \times 200$ \\
\hline \hline$L_{1}$ error of $\tilde{S}$ & 0.0021 & $6.57 \times 10^{-4}$ & $2.15 \times 10^{-4}$ & $8.75 \times 10^{-5}$ \\
\hline$L_{1}$ error of $\bar{S}$ with macrodispersion & 0.115 & 0.0696 & 0.0364 & 0.0135 \\
\hline$L_{1}$ error of $\bar{S}$ fine without macrodispersion & 0.1843 & 0.0997 & 0.0505 & 0.0191 \\
\hline
\end{tabular}

\begin{tabular}{|c||c|c|c|c|}
\hline NONLINEAR FLUX & $25 \times 25$ & $50 \times 50$ & $100 \times 100$ & $200 \times 200$ \\
\hline \hline$L_{1}$ error of $\tilde{S}$ & 0.0023 & $8.05 \times 10^{-4}$ & $2.89 \times 10^{-4}$ & $1.29 \times 10^{-4}$ \\
\hline$L_{1}$ error of $\bar{S}$ with macrodispersion & 0.116 & 0.0665 & 0.0433 & 0.0177 \\
\hline$L_{1}$ error of $\bar{S}$ fine without macrodispersion & 0.151 & 0.0805 & 0.0432 & 0.0186 \\
\hline
\end{tabular}

extra CFL-like condition to obtain a stable numerical scheme $\Delta t \leq \frac{\Delta p^{2}}{2 \nu}$, where $\nu$ is the diffusivity. In our case the diffusivity is $\int_{\text {cell }} \int_{0}^{t} \tilde{v}_{0}^{\prime}(p(\tau), \psi) \tilde{v}_{0}^{\prime}(p, \psi) d \tau d \psi$. If the macrodispersion is large this can be a very restrictive condition. To remedy this, we used an implicit discretization for the macrodispersion. This is straightforward since the problem is one-dimensional. The resulting system was solved by a tridiagonal solver very fast. Since the order of the highest derivative in the equation has increased, we require extra boundary conditions. For the computation of the macrodispersion term, we impose no flux on both boundaries of the domain.

In the upscaled algorithm, a moving mesh is used to concentrate the points of computation near the sharp front. Since the saturation equation is one dimensional in the pressure-streamline coordinates, the implementation of the moving mesh is straightforward and efficient. For the details we refer to [24]. We compare the saturation right before the breakthrough time so that the shock front is largest. For this comparison we also average the fine saturation over the coarse blocks, since the upscaled model is defined on a coarser grid. In Figures 6.2 6.3, we plot the saturation for linear and nonlinear (with $\left.\mu_{o} / \mu_{w}=5\right) f(S)$. As we see in both cases, we have very accurate representation of the saturation profile.

We proceed with a quantitative description of the error. We will distinguish between two sources of errors. We will refer to the difference between the upscaled and the exact equation as the upscaling or modeling error and to the difference between the solution of continuous upscaled equations and the solution to the numerical scheme as the discretization error. We will refer to the difference between the solutions of the continuous fine equations and the numerical scheme of the upscaled equations as the total error. To separate the upscaling error from the total error we will solve the upscaled equations on the fine grid, which is the grid on which we solve to the fine equation. We will also solve them on the coarse grid to compute the total error. The errors are computed in the $p, \psi$ frame and are relative errors. We display the upscaling error against the number of coarse cells for the computations of the previous section in Tables 6.1, 6.2, 6.3. As we see from this table that upscaling using macrodispersion decreases the upscaling errors. We also see that the effects of macrodispersion are more significant in the case of linear flux when the jump discontinuity in the saturation profile is larger.

In Tables 6.4, 6.5, 6.6, we show the total error, that is, the modeling and discretization error when we use a moving mesh to solve the saturation equation. It is interesting that the convergence of $\tilde{S}$ to $S$ is observed even though the upscaling error is larger than the numerical error of the fine solution, which is 0.02 for the linear flux and 0.002 for the nonlinear flux in the $L_{1}$ norm, as mentioned before. The reason is that the location of the moving mesh points was selected so that the points are as dense near the shock as the fine solution using the parameter $h_{\min }$. This was done to observe the upscaling error clearly and also to have similar CFL constraints on the time step, which allows a clean comparison of computational times. We compare the require CPU times in Table 6.7. We note that it took 26 units of time to interpolate one quantity from the Cartesian to the pressure-streamline frame. The upscaled solutions were computed on a $25 \times 25$ grid and the fine solution was computed on a $400 \times 400$ grid so we expect the $\bar{S}$ computations to be 256 times or more faster. The extra gain comes from a less restrictive CFL condition since we use an 

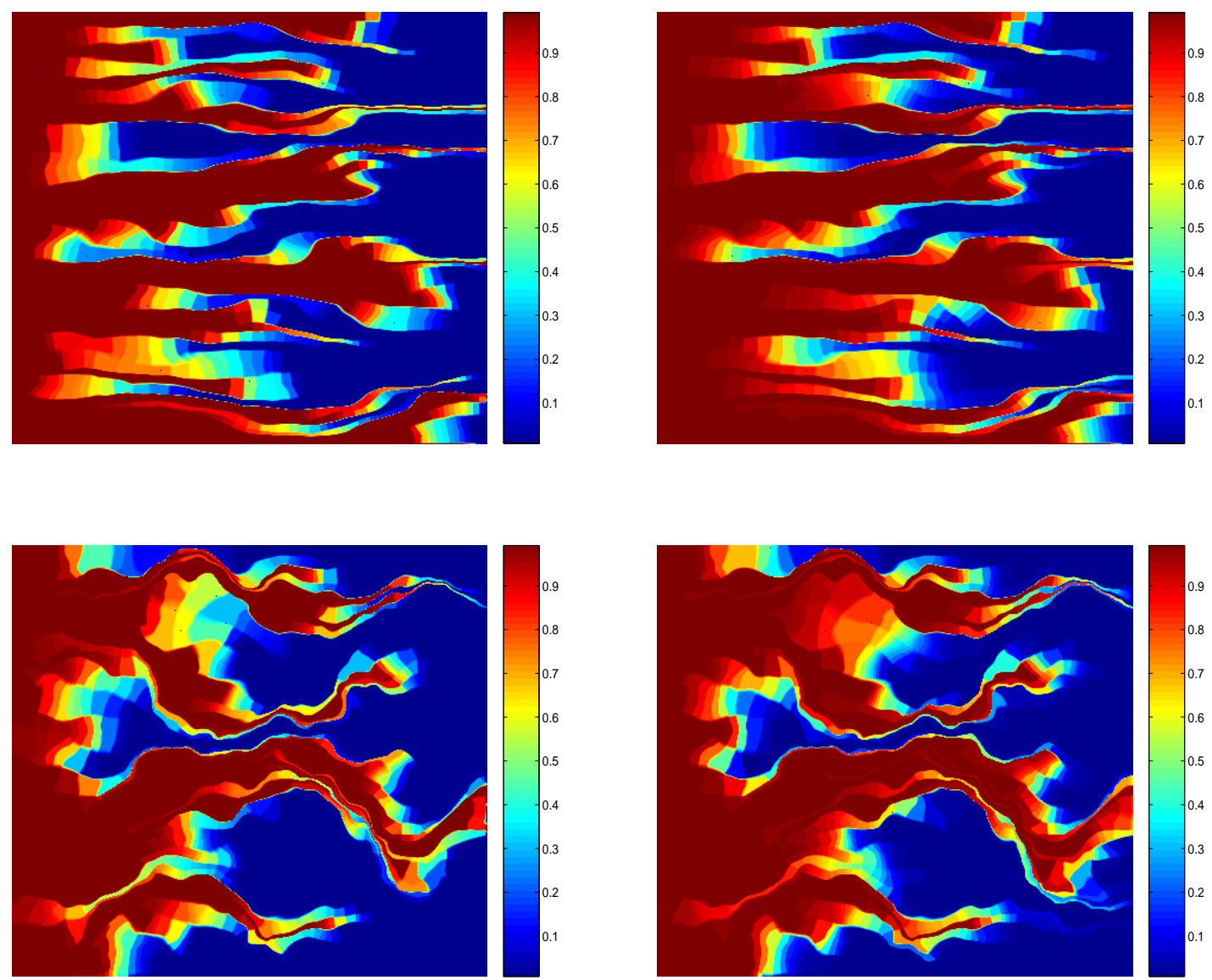

FIG. 6.2. Saturation snapshots for variogram based permeability field (top) and synthetic channelized permeability field (bottom). Linear flux is used. Left figures represent the upscaled saturation plots and the right figures represent the fine-scale saturation plots.

averaged velocity. The computations in the Cartesian frame are much slower.

The application of the proposed method to two-phase immiscible flow can be performed using the implicit pressure and explicit saturation (IMPES) framework. This procedure consists of computing the velocity and then using the velocity field in updating the saturation field. When updating the saturation field, we consider the velocity field to be time independent and we can use our upscaling procedure at each IMPES time step. First, we note that in the proposed method, the mapping is done between the current pressure-streamline and initial pressure-streamline. This mapping is nearly the identity for the cases when $\mu_{o}>\mu_{w}$. In Figure 6.4, we plot the level sets of the pressure and streamfunction at time $t=0.4$ in a Cartesian coordinate system (left plot) and in the coordinate system of the initial pressure and streamline (right plot). Clearly, the level sets are much smoother in initial pressure-streamline frame compared to Cartesian frame. This also explains the observed convergence of upscaling methods as we refined the coarse grid. In Figure 6.5, we plot the saturation snapshots right before the breakthrough. In Figure 6.6, the fractional flow is plotted. Again, the moving mesh algorithm is used to track the front separately. The convergence table is presented in Table 6.8. We see from this table that the errors decreases as first order which indicates that the pressure and saturation is smooth functions of initial pressure and streamline. 

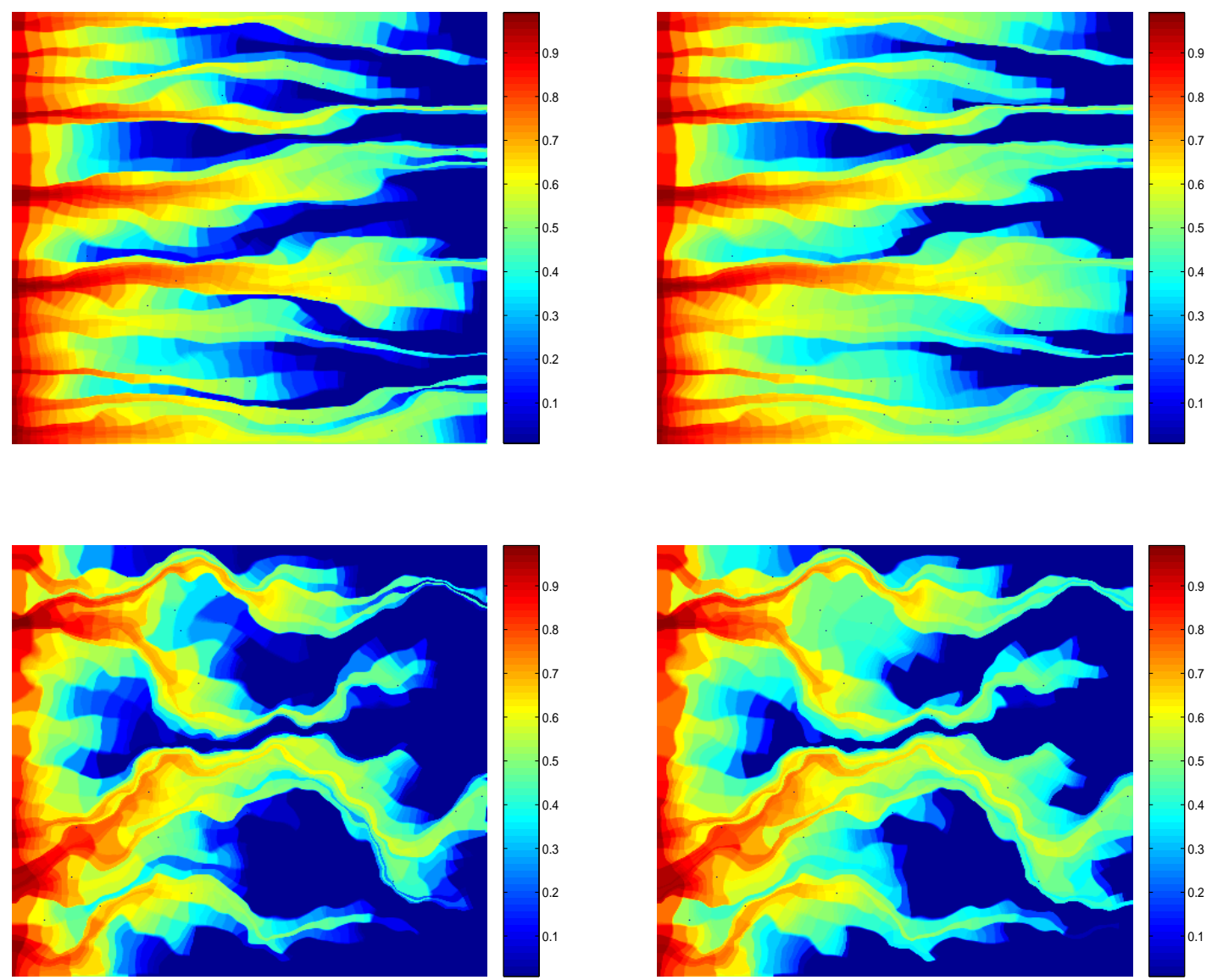

FIG. 6.3. Saturation snapshots for variogram based permeability field (top) and synthetic channelized permeability field (bottom). Nonlinear flux is used. Left figures represent the upscaled saturation plots and the right figures represent the fine-scale saturation plots.

7. Conclusions. In this paper, multiscale methods for two-phase immiscible flow using flow-based coordinate system are considered. In particular, the upscaling of convection dominated transport equation is discussed. The flow-based coordinate system allows us to simplify the scale interaction and obtain an upscaled model for transport. Further, this upscaled model is used to design a coarse-scale algorithm for two-phase flow. In our numerical methods, the shock front of the upscaled equation was resolved using a moving mesh. Numerical results show that one can achieve high accuracy using the proposed algorithms.

Though the results presented in the paper are encouraging, there are possible extensions which are currently under investigation. The extension to three dimensions is straightforward. However it fails when the coordinate transformation becomes degenerate. We conjecture (see [24]) that for most permeability fields, the regions of the flow where this occurs do not carry much fluid. It should then be possible to regularize the transformation and apply this method with only a small numerical error. Another direction of future research is the development of fast and accurate algorithms that perform interpolation from the Cartesian to pressure-streamline grid. In particular, our interest is in the development of such algorithms using coarsescale information similar to multiscale finite element methods. The latter will speed-up the interpolation computations and make the method more desirable for multi-phase flow and transport computations. 
TABLE 6.2

Upscaling error for for synthetic channelized permeability field

\begin{tabular}{|c||c|c|c|c|}
\hline LINEAR FLUX & $25 \times 25$ & $50 \times 50$ & $100 \times 100$ & $200 \times 200$ \\
\hline \hline$L_{1}$ error of $\bar{S}$ & 0.0222 & 0.0171 & 0.0122 & 0.0053 \\
\hline$L_{1}$ error of $\bar{S}$ with macrodispersion & 0.0819 & 0.0534 & 0.0333 & 0.0178 \\
\hline$L_{1}$ error of $\bar{S}$ fine without macrodispersion & 0.123 & 0.0834 & 0.0486 & 0.0209 \\
\hline
\end{tabular}

\begin{tabular}{|c||c|c|c|c|}
\hline NONLINEAR FLUX & $25 \times 25$ & $50 \times 50$ & $100 \times 100$ & $200 \times 200$ \\
\hline \hline$L_{1}$ error of $\tilde{S}$ & 0.0147 & 0.0105 & 0.0075 & 0.0040 \\
\hline$L_{1}$ error of $\bar{S}$ with macrodispersion & 0.0842 & 0.0658 & 0.0371 & 0.0207 \\
\hline$L_{1}$ error of $\bar{S}$ fine without macrodispersion & 0.119 & 0.0744 & 0.0424 & 0.0214 \\
\hline
\end{tabular}

TABLE 6.3

Upscaling error for SPE 10, layer 36

\begin{tabular}{|c||c|c|c|c|}
\hline LINEAR FLUX & $25 \times 25$ & $50 \times 50$ & $100 \times 100$ & $200 \times 200$ \\
\hline \hline$L_{1}$ error of $S$ & 0.0128 & 0.0093 & 0.0072 & 0.0042 \\
\hline$L_{1}$ error of $\bar{S}$ with macrodispersion & 0.0554 & 0.0435 & 0.0307 & 0.0176 \\
\hline$L_{1}$ error of $\bar{S}$ fine without macrodispersion & 0.123 & 0.0798 & 0.0484 & 0.0258 \\
\hline
\end{tabular}

\begin{tabular}{|c||c|c|c|c|}
\hline NONLINEAR FLUX & $25 \times 25$ & $50 \times 50$ & $100 \times 100$ & $200 \times 200$ \\
\hline \hline$L_{1}$ error of $\bar{S}$ & 0.0089 & 0.0064 & 0.0054 & 0.0033 \\
\hline$L_{1}$ error of $\bar{S}$ with macrodispersion & 0.0743 & 0.0538 & 0.0348 & 0.0189 \\
\hline$L_{1}$ error of $\bar{S}$ fine without macrodispersion & 0.0924 & 0.0602 & 0.0395 & 0.0202 \\
\hline
\end{tabular}

8. Acknowledgments. We would like to thank Dr. Victor Ginting for providing us multiscale finite volume code. We would like also to thank Dr. Yuguang Chen for providing us synthetic channelized permeability fields. The research of Y.E. is partially supported by DOE grant DE-FG02-05ER25669. T.Y.H is supported by the NSF ITR Grant ACI-0204932 and the NSF FRG grant DMS-0353838.

\section{Appendix A. Proof of Theorem 4.1.}

First, we note that the velocity bound implies that $\tilde{C}^{-1} \leq \tilde{v}_{0}(p) \leq \tilde{D}$, uniformly in $\psi, \zeta$. We transform the equations for $S^{\epsilon}(4.1)$ and $\tilde{S}(4.2)$ to the time of flight variable defined by

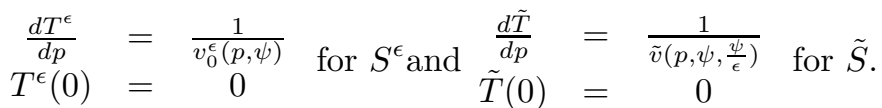

Both equations reduce to

$$
S_{t}+f(S)_{T}=0
$$

The solution to this equation is $F(t, T)$. Since the initial condition does not depend on $\epsilon$ neither does $F$. Then $S=F\left(t, T^{\epsilon}(P, \Psi)\right), \tilde{S}=F(t, \tilde{T}(P, \Psi))$. Using these expressions for the saturation we can obtain the desired estimates by following the same steps as in the linear case. When $F$ remains Lipschitz for all times we can easily obtain a pointwise estimate in terms of the Lipschitz constant $M\left\|S^{\epsilon}-\tilde{S}\right\|_{\infty}=$ $\left\|F\left(t, T^{\epsilon}\right)-F(t, \tilde{T})\right\|_{\infty} \leq M\left\|T^{\epsilon}-\tilde{T}\right\|_{\infty} \leq G \epsilon$. Otherwise we will need the time of flight bound that we derived for the linear flux that reduces here to

$$
\left|T^{\epsilon}(P)-\tilde{T}(P)\right| \leq 2 C \epsilon .
$$

We will divide the domain in regions where $F$ is Lipschitz with constant $M$ in the second variable, denoted by $A_{2}$, and shock regions, denoted by $A_{1}$, and estimate the difference of $S^{\epsilon}$ and $\tilde{S}$ in each region 
TABLE 6.4

Total error for permeability field generated using two-point geostatistics

\begin{tabular}{|c||c|c|c|c|}
\hline LINEAR FLUX & $25 \times 25$ & $50 \times 50$ & $100 \times 100$ & $200 \times 200$ \\
\hline \hline$L_{1}$ upscaling error of $\tilde{S}$ & 0.0021 & $6.57 \times 10^{-4}$ & $2.15 \times 10^{-4}$ & $8.75 \times 10^{-5}$ \\
\hline$L_{1}$ error of $\tilde{S}$ computed on coarse grid & 0.0185 & 0.0062 & 0.0019 & 0.0015 \\
\hline$L_{1}$ upscaling error of $\bar{S}$ & 0.115 & 0.0696 & 0.0364 & 0.0135 \\
\hline$L_{1}$ error of computed on coarse grid & 0.139 & 0.0779 & 0.0390 & 0.0144 \\
\hline
\end{tabular}

\begin{tabular}{|c||c|c|c|c|}
\hline NONLINEAR FLUX & $25 \times 25$ & $50 \times 50$ & $100 \times 100$ & $200 \mathrm{x} 200$ \\
\hline \hline$L_{1}$ upscaling error of $\bar{S}$ & 0.0023 & $8.05 \times 10^{-4}$ & $2.89 \times 10^{-4}$ & $1.29 \times 10^{-4}$ \\
\hline$L_{1}$ error of $\bar{S}$ computed on coarse grid & 0.0268 & 0.0099 & 0.0027 & $9.38 \times 10^{-4}$ \\
\hline$L_{1}$ upscaling error of $\bar{S}$ & 0.116 & 0.0665 & 0.0433 & 0.0177 \\
\hline$L_{1}$ error of $\bar{S}$ computed on coarse grid & 0.146 & 0.0797 & 0.0461 & 0.0184 \\
\hline
\end{tabular}

TABLE 6.5

Total error for synthetic channelized permeability field

\begin{tabular}{|c||c|c|c|c|}
\hline LINEAR FLUX & $25 \times 25$ & $50 \times 50$ & $100 \times 100$ & $200 \times 200$ \\
\hline \hline$L_{1}$ upscaling error of $\bar{S}$ & 0.0222 & 0.0171 & 0.0122 & 0.0053 \\
\hline$L_{1}$ error of $\bar{S}$ computed on coarse grid & 0.0326 & 0.0161 & 0.0107 & 0.0113 \\
\hline$L_{1}$ upscaling error of $\bar{S}$ & 0.0819 & 0.0534 & 0.0333 & 0.0178 \\
\hline$L_{1}$ error of $\bar{S}$ computed on coarse grid & 0.135 & 0.0849 & 0.0477 & 0.0274 \\
\hline
\end{tabular}

\begin{tabular}{|c||c|c|c|c|}
\hline NONLINEAR FLUX & $25 \times 25$ & $50 \times 50$ & $100 \times 100$ & $200 \times 200$ \\
\hline \hline$L_{1}$ upscaling error of $\tilde{S}$ & 0.0147 & 0.0105 & 0.0075 & 0.0040 \\
\hline$L_{1}$ error of $\bar{S}$ computed on coarse grid & 0.0494 & 0.0295 & 0.0150 & 0.0130 \\
\hline$L_{1}$ upscaling error of $\bar{S}$ & 0.0842 & 0.0658 & 0.0371 & 0.0207 \\
\hline$L_{1}$ error of $\bar{S}$ computed on coarse grid & 0.17 & 0.11 & 0.0541 & 0.0303 \\
\hline
\end{tabular}

separately. To fix the notation, let that there be $n$ discontinuities in $F(t, \cdot)$ of magnitude less than $\Delta F$, which does not have to be small, at $\left\{T=T_{i}\right\}_{i=1, \ldots, n}$. We will denote the thin strips of width $2 C \epsilon$ around the discontinuities with $A_{1}$

$$
A_{1}=\left\{T \text { such that }\left|T-T_{i}\right| \leq 2 C \epsilon \text {, for some } i=1, \ldots, n\right\}
$$

and with $A_{2}$ its complement. We selected the width of the strip based on (A.1), so that for any point $P$, if $T^{\epsilon}(P) \notin A_{1}$, then $T^{\epsilon}(P)$ and $\tilde{T}(P)$ are on the same side of any jump $T_{i}$. When $T^{\epsilon}(P) \in A_{2}, F$ is Lipschitz in the region between $T^{\epsilon}$ and $\tilde{T}$, and we can show

$$
\begin{aligned}
\int_{A_{2}}\left(S^{\epsilon}-\tilde{S}\right)^{2} d p d \psi=\int_{A_{2}}\left(F\left(t, T^{\epsilon}\right)-F(t, \tilde{T})\right)^{2} d p d \psi & \leq M^{2}\left\|T^{\epsilon}-\tilde{T}\right\|_{\infty}^{2}\left|T^{\epsilon}\left(A_{2}\right)^{-1}\right| \\
& \leq N^{2} \epsilon^{2}\left|T^{\epsilon}\left(A_{2}\right)^{-1}\right|,
\end{aligned}
$$

where we used the time of flight bound (A.1). By $\left|T^{\epsilon}\left(A_{2}\right)^{-1}\right|$ we denoted the image of $A_{2}$ under the inverse of $T^{\epsilon}(P)$. Inside the strip $A_{1}$, even though $S^{\epsilon}$ and $\tilde{S}$ differ by an $O(1)$ quantity we can use the smallness of the area of the strip to make the $L_{2}$ norm of their difference small

$$
\begin{aligned}
\int_{A_{1}}\left(S^{\epsilon}-\tilde{S}\right)^{2} d p d \psi=\int_{A_{2}}\left(F\left(t, T^{\epsilon}\right)-F(t, \tilde{T})\right)^{2} d p d \psi & \leq(\Delta S+N \epsilon)^{2}\left|T^{\epsilon}\left(A_{1}\right)^{-1}\right| \\
& \leq(\Delta S+N \epsilon)^{2} 4 C D n \epsilon .
\end{aligned}
$$

We estimated the area $\left|T^{\epsilon}\left(A_{1}\right)^{-1}\right|$ by using the definition of $A_{1}$ and the fact that the Jacobian of the transformation $T^{\epsilon}(P)^{-1}$ is $v_{0}^{\epsilon}$ and is bounded uniformly in $p, \psi$. Putting together the two estimates for regions $A_{1}$ and $A_{2}$ we obtain $\left\|S^{\epsilon}-\tilde{S}\right\|_{2} \leq G \epsilon^{1 / 2}$. Estimates in terms of the other $L_{p}$ norms follow similarly. 
TABLE 6.6

Total error for SPE10 layer 36

\begin{tabular}{|c||c|c|c|c|}
\hline LINEAR FLUX & $25 \times 25$ & $50 \times 50$ & $100 \times 100$ & $200 \times 200$ \\
\hline \hline$L_{1}$ upscaling error of $\bar{S}$ & 0.0128 & 0.0093 & 0.0072 & 0.0042 \\
\hline$L_{1}$ error of $\bar{S}$ computed on coarse grid & 0.023 & 0.0095 & 0.0069 & 0.0052 \\
\hline$L_{1}$ upscaling error of $\bar{S}$ & 0.0554 & 0.0435 & 0.0307 & 0.0176 \\
\hline$L_{1}$ error of $\bar{S}$ computed on coarse grid & 0.0683 & 0.052 & 0.0361 & 0.0205 \\
\hline
\end{tabular}

\begin{tabular}{|c||c|c|c|c|}
\hline NONLINEAR FLUX & $25 \times 25$ & $50 \times 50$ & $100 \times 100$ & $200 \times 200$ \\
\hline \hline$L_{1}$ upscaling error of $\tilde{S}$ & 0.0089 & 0.0064 & 0.0054 & 0.0033 \\
\hline$L_{1}$ error of $\bar{S}$ computed on coarse grid & 0.0338 & 0.0148 & 0.0074 & 0.0037 \\
\hline$L_{1}$ upscaling error of $\bar{S}$ & 0.0743 & 0.0538 & 0.0348 & 0.0189 \\
\hline$L_{1}$ error of $\bar{S}$ computed on coarse grid & 0.115 & 0.0720 & 0.0406 & 0.0204 \\
\hline
\end{tabular}

TABLE 6.7

Computational cost

\begin{tabular}{|c||c|c|c|c|}
\hline & fine $x . y$ & fine $p, \psi$ & $\tilde{S}$ & $\bar{S}$ \\
\hline \hline layered, linear flux & 5648 & 257 & 9 & 1 \\
\hline layered, nonlinear flux & 14543 & 945 & 28 & 4 \\
\hline percolation, linear flux & 8812 & 552 & 12 & 1 \\
\hline percolation, nonlinear flux & 23466 & 579 & 12 & 1 \\
\hline SPE10 36, linear flux & 40586 & 1835 & 34 & 2 \\
\hline SPE10 36, nonlinear flux & 118364 & 7644 & 25 & 2 \\
\hline
\end{tabular}

\section{REFERENCES}

[1] J. AARNES, On the use of a mixed multiscale finite element method for greater flexibility and increased speed or improved accuracy in reservoir simulation, SIAM MMS, 2 (2004), pp. 421-439.

[2] T. Arbogast, Implementation of a locally conservative numerical subgrid upscaling scheme for two-phase Darcy flow, Comput. Geosci., 6 (2002), pp. 453-481. Locally conservative numerical methods for flow in porous media.

[3] I. BabušKa, G. CAloz, And E. Osborn, Special finite element methods for a class of second order elliptic problems with rough coefficients, SIAM J. Numer. Anal., 31 (1994), pp. 945-981.

[4] I. BABUs̆KA AND E. OsBorn, Generalized finite element methods: Their performance and their relation to mixed methods, SIAM J. Numer. Anal., 20 (1983), pp. 510-536.

[5] A. Bourgeat And A. Mikelić, Homogenization of two-phase immiscible flows in a one-dimensional porous medium, Asymptotic Anal., 9 (1994), pp. 359-380.

[6] F. BREzZI, Interacting with the subgrid world, in Numerical analysis 1999 (Dundee), Chapman \& Hall/CRC, Boca Raton, FL, 2000, pp. 69-82.

[7] Y. Chen And L. J. Durlofsky, Adaptive coupled local-global upscaling for general flow scenarios in heterogeneous formations, Transport in Porous media. to appear.

[8] Z. Chen And T. Y. Hou, A mixed multiscale finite element method for elliptic problems with oscillating coefficients, Math. Comp., 72 (2002), pp. 541-576 (electronic).

[9] M. Christie And M. Blunt, Tenth spe comparative solution project: A comparison of upscaling techniques, SPE Reser. Eval. Eng., 4 (2001), pp. 308-317.

[10] W. E, Homogenization of linear and nonlinear transport equations, Comm. Pure Appl. Math., XLV (1992), pp. 301-326.

[11] Y. Efendiev, V. Ginting, T. Hou, And R. Ewing, Accurate multiscale finite element methods for two-phase flow simulations. Submitted.

[12] Y. Efendiev, T. Hou, And V. Ginting, Multiscale finite element methods for nonlinear problems and their applications, Comm. Math. Sci., 2 (2004), pp. 553-589.

[13] Y. R. Efendiev And L. J. DuRlofsky, Numerical modeling of subgrid heterogeneity in two phase flow simulations, Water Resour. Res., 38(8) (2002), p. 1128.

[14] Y. R. Efendiev, L. J. Durlofsky, And S. H. Lee, Modeling of subgrid effects in coarse scale simulations of transport in heterogeneous porous media, Water Resour. Res., 36 (2000), pp. 2031-2041.

[15] Y. R. Efendiev And B. Popov, On homogenization of nonlinear hyperbolic equations, Communications on Pure and Applied Analysis, 4(2) (2005), pp. 295-309. 

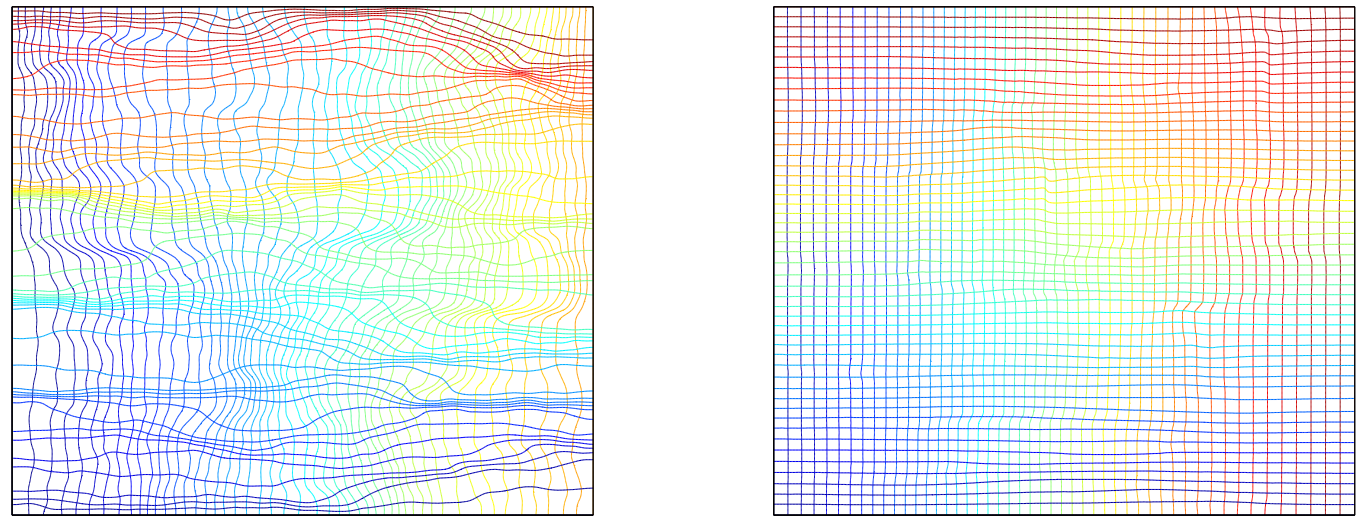

Fig. 6.4. Left: Pressure and streamline function at time $t=0.4$ in Cartesian frame. Right: pressure and streamline function at time $t=0.4$ in initial pressure-streamline frame.
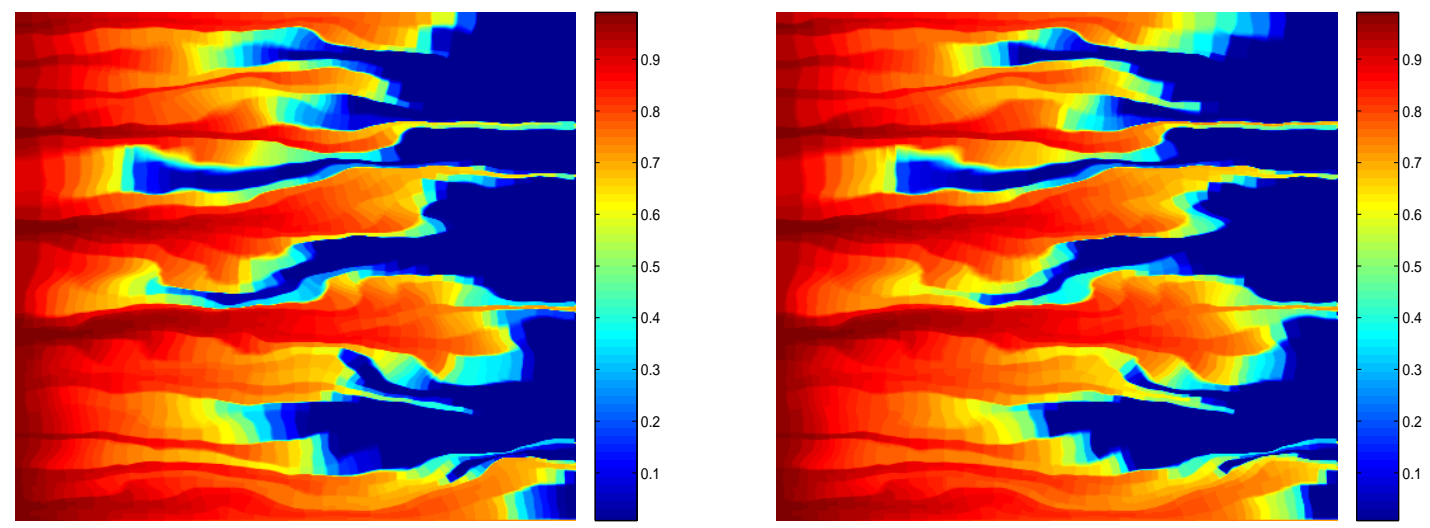

FIG. 6.5. Left: Saturation plot obtained using coarse-scale model. Right: The fine-scale saturation plot. Both plots are on coarse grid. Variogram based permeability field is used. $\mu_{o} / \mu_{w}=5$.

TABLE 6.8

Convergence of the upscaling method for two-phase flow for variogram based permeability field

\begin{tabular}{|c||c|c|c|}
\hline with $\tilde{S}$ & $50 \mathrm{x} 50$ & $100 \mathrm{x} 100$ & $200 \mathrm{x} 200$ \\
\hline \hline$L_{2}$ pressure error at $t=\frac{3 T_{\text {final }}}{4}$ & 0.0014 & 0.007 & 0.004 \\
\hline$L_{2}$ velocity error at $t=\frac{3 T_{\text {final }}}{4}$ & 0.0235 & 0.0137 & 0.0072 \\
\hline$L_{1}$ saturation error $t=T_{\text {final }}$ & 0.0105 & 0.0052 & 0.0027 \\
\hline & \multicolumn{3}{|l}{} \\
\hline
\end{tabular}

\begin{tabular}{|c||c|c|c|}
\hline with $\bar{S}$ & $50 \times 50$ & $100 \times 100$ & $200 \times 200$ \\
\hline \hline$L_{2}$ pressure error at $t=\frac{3 T_{\text {final }}}{4}$ & 0.0046 & 0.0021 & 0.0008 \\
\hline$L_{2}$ velocity error at $t=\frac{3 T_{\text {final }}}{4}$ & 0.0530 & 0.0335 & 0.0246 \\
\hline$L_{1}$ saturation error $t=T_{\text {final }}$ & 0.0546 & 0.0294 & 0.0134 \\
\hline
\end{tabular}




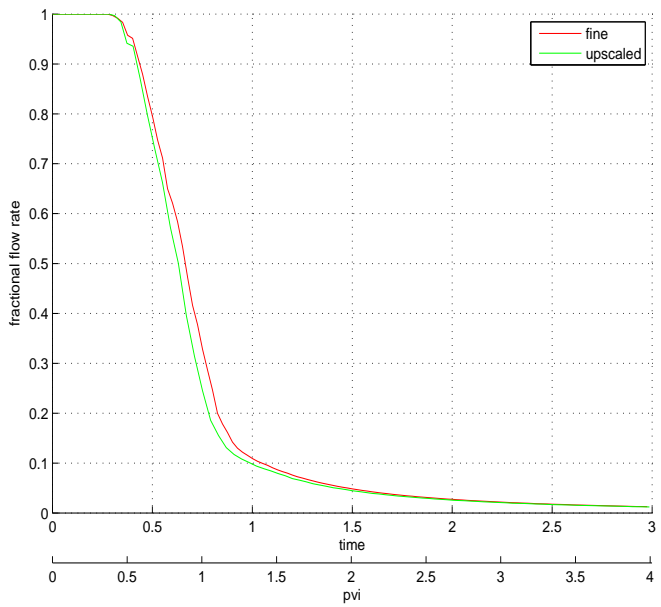

FIG. 6.6. Comparison of fractional flow for coarse- and fine-scale models. Variogram based permeability field is used. $\mu_{o} / \mu_{w}=5$.

[16] T. Y. Hou, A. Westhead, And D. P. Yang, A framework for modeling subgrid effects for two-phase flows in porous media. submitted to SIAM Multiscale Modeling and Simulation.

[17] T. Y. Hou AND X. H. WU, A multiscale finite element method for elliptic problems in composite materials and porous media, Journal of Computational Physics, 134 (1997), pp. 169-189.

[18] T. Y. Hou AND X. XIN, Homogenization of linear transport equations with oscillatory vector fields, SIAM J. Appl. Math., 52 (1992), pp. 34-45.

[19] T. Hughes, G. Feijoo, L. Mazzei, And J. Quincy, The variational multiscale method - a paradigm for computational mechanics, Comput. Methods Appl. Mech. Engrg, 166 (1998), pp. 3-24.

[20] P. Jenny, S. H. Lee, And H. TChelePI, Multi-scale finite volume method for elliptic problems in subsurface flow simulation, J. Comput. Phys., 187 (2003), pp. 47-67.

[21] — Adaptive multi-scale finite volume method for multi-phase flow and transport in porous media, Multiscale Modeling and Simulation, 3 (2005), pp. 30-64.

[22] A.-M. Matache and C. Schwab, Homogenization via p-FEM for problems with microstructure, in Proceedings of the Fourth International Conference on Spectral and High Order Methods (ICOSAHOM 1998) (Herzliya), vol. 33, 2000, pp. $43-59$.

[23] G. Sangalli, Capturing small scales in elliptic problems using a residual-free bubbles finite element method, Multiscale Model. Simul., 1 (2003), pp. 485-503 (electronic).

[24] T. Strinopoulos, Upscaling of immiscible two-phase flows in an adaptive frame, $\mathrm{PhD}$ thesis, California Institute of Technology, Pasadena, 2005.

[25] L. TARTAR, Nonlocal effects induced by homogenization, PDE and calculus of variations, (1989), pp. 925-938. Boston.

[26] X. Wen, L. Durlofsky, And M. Edwards, Upscaling of channel systems in two dimensions using flow-based grids, Transport in Porous Media, 51 (2003), pp. 343-366.

[27] A. Westhead, Upscaling two-phase flows in porous media, PhD thesis, California Institute of Technology, Pasadena, 2005. 